\title{
Topological Derivatives in Plane Elasticity
}

\author{
Jan Sokołowski ${ }^{1}$ and Antoni Żochowski ${ }^{2, \star}$ \\ 1 Institut Elie Cartan, Laboratoire de Mathématiques, \\ Université Henri Poincaré Nancy 1, \\ B.P. 239, 54506 Vandoeuvre lés Nancy Cedex, France \\ Jan. Sokolowski@iecn.u-nancy. fr \\ 2 Systems Research Institute of the Polish Academy of Sciences, \\ ul. Newelska 6, 01-447 Warszawa, Poland \\ zochowsk@ibspan.waw.p1
}

\begin{abstract}
We present a method for construction of the topological derivatives in plane elasticity. It is assumed that a hole is created in the subdomain of the elastic body which is filled out with isotropic material. The asymptotic analysis of elliptic boundary value problems in singularly perturbed geometrical domains is used in order to derive the asymptotics of the shape functionals depending on the solutions to the boundary value problems. Our method allows for the asymptotic expansions of arbitrary order, since the explicit solutions to the boundary value problems are obtained by the method of elastic potentials. Some numerical results are presented to show the applicability of the proposed method in numerical analysis of elliptic problems.
\end{abstract}

\section{Introduction}

One of the most important applications of the topological derivatives of shape functionals is elasticity, in particular in the fields of optimal design in structural mechanics and the numerical solution for inverse problems of detection of small imperfections. The mathematical theory of asymptotic analysis of elliptic boundary value problems in singularly perturbed domains, is considered in [6] and [10]. The method of compound asymptotic expansions in the framework of the asymptotic analysis leads to the asymptotic expansions of solutions and to the topological derivatives of the shape functionals as it is described in details, e.g., in the paper [12] for boundary value problems of linearized elasticity. The concept of topological derivatives of shape functionals [18] is derived in the framework of the method of compound asymptotic expansions [10], one of the techniques used in the asymptotic analysis of the boundary value problems in singularly perturbed geometrical domains. The so-called truncation method is described, e.g., in [9] (see [3] for further developments). The asymptotic analysis in impedance imaging and the theory of composite materials can be found, e.g., in [1].

We present here the results on asymptotics of the shape functionals for the specific class of the elliptic boundary value problems. Let there be given an elastic body which occupies the reference domain $\Omega \subset \mathbb{R}^{d}, d=2,3$, with the material properties defined by the Hooke's tensor $\mathscr{C}_{i j k l}, i, j, k, l=1, \ldots, d$. We assume that there is a ball

\footnotetext{
* The research is supported by the grant N51402132/3135 of the Polish Ministry of Education.
}

A. Korytowski et al. (Eds.): System Modeling and Optimization, IFIP AICT 312, pp. 459-475, 2009.

(c) IFIP International Federation for Information Processing 2009 
$B_{R}(x) \subset \Omega, R>0$, with the center $x \in \Omega$, filled with an isotropic material characterized by its Lame coefficients $\lambda, \mu$. We investigate the asymptotics for $\rho \rightarrow 0$ of the displacement and the stress fields in the body $\Omega_{\rho}=\Omega \backslash \overline{B_{\rho}(x)}$ due to the creation of a small hole $B_{\rho}(x) \subset B_{R}(x)$ of the radius $R>\rho \rightarrow 0$. We also perform the asymptotic analysis of some shape functionals depending on the solution of the elasticity boundary value problems in $\Omega_{\rho}=\Omega \backslash \overline{B_{\rho}(x)}$ for $\rho \rightarrow 0$. It seems that the imposed condition on the isotropy of $B_{R}(x)$ cannot be avoided since for the specific application of the existing methods of asymptotic analysis we need the knowledge of fundamental solution of the elliptic operator in the region $B_{R}(x)$. In order to obtain the required asymptotics in the whole domain we employ [21] a domain decomposition technique combined with the fine analysis of the properties of the Steklov-Poincaré operator $\mathscr{A}_{\rho}, \rho \geq 0$, defined in the ball $B_{R}(x)$ as well as in the ring $C(R, \rho)=B_{R}(x) \backslash \overline{B_{\rho}(x)}$.

The paper contains the complete mathematical tools which are used to derive the form of topological derivatives for the specific class of composite elastic materials in two spatial dimensions.

\section{Topological Derivatives of Shape Functionals in Isotropic Elasticity}

We are going to present the results which can be obtained for 2D boundary value problems of linear elasticity. The results for 3D are not in the same explicit form. The same type of results on topological derivatives is derived for the contact problems by means of the asymptotic analysis combined with the domain decomposition technique [21].

We briefly introduce the concept of the topological derivative for an arbitrary shape functional. The topological derivative denoted by $\mathscr{T}_{\Omega}$ of a shape functional $\mathscr{J}(\Omega)$ is introduced in [18] in order to characterize the infinitesimal variation of $\mathscr{J}(\Omega)$ with respect to the infinitesimal variation of the topology of the domain $\Omega$. The topological derivative allows us to derive the new optimality condition in the interior of an optimal domain, if such a domain exists and if the shape functional under studies admits the topological derivatives, for the shape optimization problem:

$$
\mathscr{J}\left(\Omega^{*}\right)=\inf _{\Omega} \mathscr{J}(\Omega) .
$$

The optimal domain $\Omega^{*}$ is characterized by the first order condition [17] defined on the boundary of the optimal domain $\Omega^{*}, d J\left(\Omega^{*} ; V\right) \geq 0$ for all admissible vector fields $V$, and by the following optimality condition defined in the interior of the domain $\Omega^{*}$ :

$$
\mathscr{T}_{\Omega^{*}}(x) \geq 0 \text { in } \Omega^{*} .
$$

The other use of the topological derivative is connected with approximating the influence of the holes in the domain on the values of integral functionals of solutions, which allows us, e.g., to solve a class of shape inverse problems.

In general terms the notion of the topological derivative (TD) has the following meaning. Assume that $\Omega \subset \mathbb{R}^{N}$ is an open set and that there is given a shape functional

$$
\mathscr{J}: \Omega \backslash K \rightarrow \mathbb{R}
$$


for any compact subset $K \subset \bar{\Omega}$. We denote by $B_{\rho}(x), x \in \Omega$, the ball of radius $\rho>0$, $B_{\rho}(x)=\left\{y \in \mathbb{R}^{N} \mid\|y-x\|<\rho\right\}, \overline{B_{\rho}(x)}$ is the closure of $B_{\rho}(x)$, and assume that there exists the following limit

$$
\mathfrak{T}(x)=\lim _{\rho \downarrow 0} \frac{\mathscr{J}\left(\Omega \backslash \overline{B_{\rho}(x)}\right)-\mathscr{J}(\Omega)}{\left|\overline{B_{\rho}(x)}\right|} .
$$

The function $\mathfrak{T}(x), x \in \Omega$, is called the topological derivative of $\mathscr{J}(\Omega)$, and provides the information on the infinitesimal variation of the shape functional $\mathscr{J}$ if a small hole is created at $x \in \Omega$. This definition is suitable for Neumann-type boundary conditions on $\partial B_{\rho}$.

In many cases this characterization is constructive [5|2|3|r|12 14 [15], i.e. TD can be evaluated for shape functionals depending on solutions of partial differential equations defined in the domain $\Omega$.

\subsection{Problem Setting for Elasticity Systems}

We introduce the elasticity system in a form convenient for the evaluation of topological derivatives. Let us consider the elasticity equations in $\mathbb{R}^{N}$, where $N=2$ for $2 \mathrm{D}$ and $N=3$ for $3 \mathrm{D}$,

$$
\left\{\begin{aligned}
\operatorname{div} \sigma(u) & =0 \text { in } \Omega \\
u & =g \text { on } \Gamma_{D} \\
\sigma(u) n & =T \text { on } \Gamma_{N}
\end{aligned}\right.
$$

and the same system in the domain with the spherical cavity $B_{\rho}\left(x_{0}\right) \subset \Omega$ centered at $x_{0} \in \Omega, \Omega_{\rho}=\Omega \backslash \overline{B_{\rho}\left(x_{0}\right)}$,

$$
\left\{\begin{aligned}
\operatorname{div} \sigma_{\rho}\left(u_{\rho}\right)=0 & \text { in } \Omega_{\rho} \\
u_{\rho}=g & \text { on } \Gamma_{D} \\
\sigma_{\rho}\left(u_{\rho}\right) n=T & \text { on } \Gamma_{N} \\
\sigma_{\rho}\left(u_{\rho}\right) n=0 & \text { on } \partial B_{\rho}\left(x_{0}\right)
\end{aligned}\right.
$$

where $n$ is the unit outward normal vector on $\partial \Omega_{\rho}=\partial \Omega \cup \partial B_{\rho}\left(x_{0}\right)$. Assuming that $0 \in \Omega$, we can consider the case $x_{0}=0$.

Here $u$ and $u_{\rho}$ denote the displacement vectors fields, $g$ is a given displacement on the fixed part $\Gamma_{D}$ of the boundary, $t$ is a traction prescribed on the loaded part $\Gamma_{N}$ of the boundary. In addition, $\sigma$ is the Cauchy stress tensor given, for $\xi=u$ (eq. 5] or $\xi=u_{\rho}$ (eq. 6), by

$$
\sigma(\xi)=D \nabla^{s} \xi
$$

where $\nabla^{s}(\xi)$ is the symmetric part of the gradient of vector field $\xi$, that is

$$
\nabla^{s}(\xi)=\frac{1}{2}\left(\nabla \xi+\nabla \xi^{T}\right)
$$

and $D$ is the elasticity tensor,

$$
D=2 \mu I+\lambda(I \otimes I),
$$


with

$$
\mu=\frac{E}{2(1+v)}, \lambda=\frac{v E}{(1+v)(1-2 v)} \text { and } \lambda=\lambda^{*}=\frac{v E}{1-v^{2}},
$$

$E$ being the Young's modulus, $v$ the Poisson's ratio and $\lambda^{*}$ the particular case for plane stress. In addition, I and II respectively are the second and fourth order identity tensors. Thus, the inverse of $D$ is

$$
D^{-1}=\frac{1}{2 \mu}\left[I-\frac{\lambda}{2 \mu+N \lambda}(I \otimes I)\right] .
$$

The first shape functional under consideration depends on the displacement field,

$$
J_{u}(\rho)=\int_{\Omega_{\rho}} F\left(u_{\rho}\right) d \Omega, F\left(u_{\rho}\right)=\left(H u_{\rho} \cdot u_{\rho}\right)^{p},
$$

where $F$ is a $C^{2}$ function. It is also useful for further applications in the framework of elasticity to introduce the yield functional of the form

$$
J_{\sigma}(\rho)=\int_{\Omega_{\rho}} S \sigma\left(u_{\rho}\right) \cdot \sigma\left(u_{\rho}\right) d \Omega,
$$

where $S$ is an isotropic fourth-order tensor. Isotropicity means here that $S$ may be expressed as follows

$$
S=2 m I I+l(I \otimes I),
$$

where $l, m$ are real constants. Their values may vary for particular yield criteria. The following assumption assures that $J_{u}, J_{\sigma}$ are well defined for solutions of the elasticity system.

(CONDITION A) The domain $\Omega$ has piecewise smooth boundary, which may have reentrant corners with $\alpha<2 \pi$ created by the intersection of two planes. In addition, $g$, $t$ must be compatible with $u \in H^{1}\left(\Omega ; \mathbb{R}^{N}\right)$.

The interior regularity of $u$ in $\Omega$ is determined by the regularity of the right hand side of the elasticity system. For simplicity the following notation is used for functional spaces,

$$
\begin{gathered}
H_{g}^{1}\left(\Omega_{\rho}\right)=\left\{\psi \in\left[H^{1}\left(\Omega_{\rho}\right)\right]^{N} \mid \psi=g \text { on } \Gamma_{D}\right\}, \\
H_{\Gamma_{D}}^{1}\left(\Omega_{\rho}\right)=\left\{\psi \in\left[H^{1}\left(\Omega_{\rho}\right)\right]^{N} \mid \psi=0 \text { on } \Gamma_{D}\right\}, \\
H_{\Gamma_{D}}^{1}(\Omega)=\left\{\psi \in\left[H^{1}(\Omega)\right]^{N} \mid \psi=0 \text { on } \Gamma_{D}\right\},
\end{gathered}
$$

here we use the convention that, e.g., $H_{g}^{1}\left(\Omega_{\rho}\right)$ stands for the Sobolev space of vector functions $\left[H_{g}^{1}\left(\Omega_{\rho}\right)\right]^{N}$.

The weak solutions to the elasticity systems are defined in the standard way.

Find $u_{\rho} \in H_{g}^{1}\left(\Omega_{\rho}\right)$ such that, for every $\phi \in H_{\Gamma_{D}}^{1}(\Omega)$,

$$
\int_{\Omega_{\rho}} D \nabla^{s} u_{\rho} \cdot \nabla^{s} \phi d \Omega=\int_{\Gamma_{N}} T \cdot \phi d S .
$$


We introduce the adjoint state equations in order to simplify the form of shape derivatives of functionals $J_{u}, J_{\sigma}$. For the functional $J_{u}$ they take on the variational form: Find $w_{\rho} \in H_{I_{D}}^{1}\left(\Omega_{\rho}\right)$,

$$
\int_{\Omega_{\rho}} D \nabla^{s} w_{\rho} \cdot \nabla^{s} \phi d \Omega=-\int_{\Omega_{\rho}} F_{u}^{\prime}\left(u_{\rho}\right) \cdot \phi d \Omega,
$$

for every $\phi \in H_{\Gamma_{D}}^{1}(\Omega)$, whose Euler-Lagrange equation reads

$$
\left\{\begin{aligned}
\operatorname{div} \sigma_{\rho}\left(w_{\rho}\right) & =F_{u}^{\prime}\left(u_{\rho}\right) & & \text { in } \Omega_{\rho} \\
w_{\rho} & =0 & & \text { on } \Gamma_{D} \\
\sigma_{\rho}\left(w_{\rho}\right) n & =0 & & \text { on } \Gamma_{N} \\
\sigma_{\rho}\left(w_{\rho}\right) n & =0 & & \text { on } \partial B_{\rho}\left(x_{0}\right)
\end{aligned}\right.
$$

while $v_{\rho} \in H_{\Gamma_{D}}^{1}\left(\Omega_{\rho}\right)$ is the adjoint state for $J_{\sigma}$ and satisfies for all test functions $\phi \in$ $H_{\Gamma_{D}}^{1}(\Omega)$ the following integral identity:

$$
\int_{\Omega_{\rho}} D \nabla^{s} v_{\rho} \cdot \nabla^{s} \phi d \Omega=-2 \int_{\Omega_{\rho}} D S \sigma\left(u_{\rho}\right) \cdot \nabla^{s} \phi d \Omega,
$$

whose associated Euler-Lagrange equation becomes

$$
\left\{\begin{aligned}
\operatorname{div} \sigma_{\rho}\left(v_{\rho}\right) & =-2 \operatorname{div}\left(D S \sigma_{\rho}\left(u_{\rho}\right)\right) & & \text { in } \Omega_{\rho} \\
v_{\rho} & =0 & & \text { on } \Gamma_{D} \\
\sigma_{\rho}\left(v_{\rho}\right) n & =-2 D S \sigma_{\rho}\left(u_{\rho}\right) n & & \text { on } \Gamma_{N} \\
\sigma_{\rho}\left(v_{\rho}\right) n & =-2 D S \sigma_{\rho}\left(u_{\rho}\right) n & & \text { on } S_{\rho}\left(x_{0}\right)=\partial B_{\rho}\left(x_{0}\right)
\end{aligned}\right.
$$

Remark 1. We observe that $D S$ can be written as

$$
D S=4 \mu m I I+\gamma(I \otimes I)
$$

where

$$
\gamma=\lambda l N+2(\lambda m+\mu l) .
$$

Thus, when $\gamma=0$, the boundary condition on $\partial B_{\rho}\left(x_{0}\right)$ in equation (22) becomes homogeneous and the yield criteria must satisfy the constraint

$$
\frac{m}{l}=-\left(\frac{\mu}{\lambda}+\frac{N}{2}\right),
$$

which is satisfied for the energy shape functional. In this particular case, tensor $S$ is given by

$$
S=\frac{1}{2} D^{-1} \Rightarrow \gamma=0 \text { and } 2 m+l=\frac{1}{2 E},
$$

which implies that the adjoint solution associated to $J_{\sigma}$ can be explicitly obtained, such that $v_{\rho}=-\left(u_{\rho}-g\right)$. 


\subsection{Topological Derivatives in 2D Elasticity}

We recall here the results derived in [18] for the 2D case. The principal stresses associated with the displacement field $u$ are denoted by $\sigma_{I}(u), \sigma_{I I}(u)$, the trace of the stress tensor $\sigma(u)$ is denoted by $\operatorname{tr} \sigma(u)=\sigma_{I}(u)+\sigma_{I I}(u)$. The shape functionals $J_{u}, J_{\sigma}$ are defined in the same way as presented before, with the tensor $S$ isotropic (that is similar to $D$ ). The weak solutions to the elasticity system as well as adjoint equations are defined in standard way. Then, from the expansions presented in the Appendix, we may formulate the following result [18]:

Theorem 1. The expressions for the topological derivatives of the functionals $J_{u}, J_{\sigma}$ have the form

$$
\begin{aligned}
\mathscr{T} J_{u}\left(x_{0}\right)=- & {\left[F(u)+\frac{1}{E}\left(a_{u} a_{w}+2 b_{u} b_{w} \cos 2 \delta\right)\right]_{x=x_{0}} } \\
=- & {\left[F(u)+\frac{1}{E}(4 \sigma(u) \cdot \sigma(w)-\operatorname{tr} \sigma(u) \operatorname{tr} \sigma(w))\right]_{x=x_{0}} } \\
\mathscr{T} J_{\sigma}\left(x_{0}\right)=- & {\left[\eta\left(a_{u}^{2}+2 b_{u}^{2}\right)+\frac{1}{E}\left(a_{u} a_{v}+2 b_{u} b_{v} \cos 2 \delta\right)\right]_{x=x_{0}} } \\
=- & {\left[\eta\left(4 \sigma(u) \cdot \sigma(u)-(\operatorname{tr} \sigma(u))^{2}\right)\right.} \\
& \left.+\frac{1}{E}(4 \sigma(u) \cdot \sigma(v)-\operatorname{tr} \sigma(u) \operatorname{tr} \sigma(v))\right]_{x=x_{0}}
\end{aligned}
$$

Some of the terms in 27), (28) require explanation. According to equation (24) for $N=2$, constant $\eta$ is given by

$$
\eta=l+2\left(m+\gamma \frac{v}{E}\right)
$$

Furthermore, we denote

$$
\begin{array}{ll}
a_{u}=\sigma_{I}(u)+\sigma_{I I}(u), & b_{u}=\sigma_{I}(u)-\sigma_{I I}(u), \\
a_{w}=\sigma_{I}(w)+\sigma_{I I}(w), & b_{w}=\sigma_{I}(w)-\sigma_{I I}(w), \\
a_{v}=\sigma_{I}(v)+\sigma_{I I}(v), & b_{v}=\sigma_{I}(v)-\sigma_{I I}(v) .
\end{array}
$$

$\delta$ denotes the angle between principal stress directions for displacement fields $u$ and $w$ in (27), and for displacement fields $u$ and $v$ in (28).

Remark 2. For the energy stored in a 2D elastic body, tensor $S$ is given by eq. (26), $\gamma=0$ and $\eta=1 /(2 E)$. Thus, since $v=-(u-g)$, we obtain the following well-known result

$$
\mathscr{T} J_{\sigma}\left(x_{0}\right)=\frac{1}{2 E}\left[4 \sigma(u) \cdot \sigma(u)-(\operatorname{tr} \sigma(u))^{2}\right]_{x=x_{0}} .
$$




\section{Topological Derivatives for Contact Problems}

In order to describe the domain decomposition method applied to the asymptotic analysis, and introduce the Steklov-Poincaré operators for the rings $C(R, \rho), \rho \geq 0$, we present the related results for the two dimensional frictionless contact problems. Such problems are non smooth, therefore, in general, only the first term of the exterior asymptotic expansion of solutions can be derived. However, this leads to the topological derivatives of some shape functionals. We change the notation, compared to the previous sections, in particular $\mathbf{u}$ stands now for the displacement vector, and $\sigma(\mathbf{u})$ is the corresponding stress tensor.

We consider the isotropic two dimensional elasticity problem in plane stress formulation, the isotropy is in fact required only in the vicinity of a small hole. On a part $\Gamma_{u}$ of $\partial \Omega$ we assume that the body is clamped $\mathbf{u}=0$, the part $\Gamma_{g}$ is loaded $\sigma(\mathbf{u}) \cdot \mathbf{n}=\mathbf{g}$ and on the part $\Gamma_{c}$ there is the frictionless contact

$$
\begin{aligned}
u_{n} & \geq 0, & & \sigma_{n} \leq 0, \\
\sigma_{n} u_{n} & =0, & & \sigma_{\tau}=\sigma \cdot \mathbf{n}-\sigma_{n} \mathbf{n}=0 .
\end{aligned}
$$

Here $u_{n}=u_{i} n_{i}, \sigma_{n}=n_{i} \sigma_{i j} n_{j}, \sigma . \mathbf{n}=\left\{\sigma_{i j} n_{j}\right\}_{i=1,2}$. We define also the ring $C(R, \rho)=$ $B(R) \backslash \overline{B(\rho)}$ with $R>\rho$ and such that $B(R) \subset \Omega$, as well as $\Omega(r)=\Omega \backslash \overline{B(r)}$.

For such a problem it is impossible to evaluate topological derivatives of shape functionals by means of adjoint variables without additional assumptions on the strict complementarity type for the unknown solution. Therefore, we propose a method for computing the perturbation, caused by the hole $B(\rho)$, of the solution itself.

The bilinear form corresponding to the elastic energy may be written as

$$
a(\rho ; \mathbf{u}, \mathbf{v})=\frac{1}{2} \int_{\Omega(\rho)} \sigma(\mathbf{u}): \varepsilon(\mathbf{v}) d x
$$

$\left(\sigma: \varepsilon=\sigma_{i j} \varepsilon_{i j}\right)$ for $\mathbf{u}, \mathbf{v} \in \mathbf{H}^{1}(\Omega)$ and the work of external forces is

$$
L(\mathbf{u})=\int_{\Gamma_{g}} \mathbf{u}^{\top} \mathbf{g} d s .
$$

The method of the domain decomposition type is based on the analysis of the SteklovPoincaré operator $\mathscr{A}_{\rho}$ defined in the following way. Consider the boundary value problem

$$
\mathscr{L} \mathbf{w}=0 \text { in } C(R, \rho), \sigma_{n}(\mathbf{w})=0 \text { on } \partial B(\rho), \mathbf{w}=\mathbf{v} \text { on } \partial B(R) .
$$

Then we set

$$
\mathscr{A}_{\rho} \mathbf{v}=\sigma_{n}(\mathbf{w}) \text { on } \partial B(R)
$$

Thus $\mathscr{A}_{\rho}$ is a mapping

$$
\mathscr{A}_{\rho}: \mathbf{H}^{1 / 2}(\partial B(R)) \mapsto \mathbf{H}^{-1 / 2}(\partial B(R)) .
$$

It can be demonstrated constructively that

$$
\mathscr{A}_{\rho}=\mathscr{A}_{0}+\rho^{2} \mathscr{A}_{1}+\rho^{4} \mathscr{A}_{2}+\ldots
$$


in the linear operator norm corresponding to [37). Using this notation we have

$$
a(\rho ; u, u)=\frac{1}{2} \int_{\Omega(R)} \sigma(\mathbf{u}): \varepsilon(\mathbf{u}) d x+\frac{1}{2} \int_{C(R, \rho)} \sigma(\mathbf{u}): \varepsilon(\mathbf{u}) d x
$$

as well as

$$
\begin{aligned}
\frac{1}{2} \int_{C(R, \rho)} \sigma(\mathbf{u}): \varepsilon(\mathbf{u}) d x & =\frac{1}{2}\left\langle\mathscr{A}_{\rho} \mathbf{u}, \mathbf{u}\right\rangle_{\partial B(R)} \\
& =\frac{1}{2}\left\langle\mathscr{A}_{0} \mathbf{u}, \mathbf{u}\right\rangle_{\partial B(R)}+\frac{1}{2} \rho^{2}\left\langle\mathscr{A}_{1} \mathbf{u}, \mathbf{u}\right\rangle_{\partial B(R)}+\mathscr{R}(\mathbf{u}, \mathbf{u})
\end{aligned}
$$

where $\mathscr{R}(\mathbf{u}, \mathbf{u})$ is of the order $O\left(\rho^{4}\right)$ on bounded sets in $\mathbf{H}^{1 / 2}(\partial B(R))$. With $\mathscr{A}_{1}$ we associate the bilinear form

$$
b(\mathbf{u}, \mathbf{v})=\frac{1}{2}\left\langle\mathscr{A}_{1} \mathbf{u}, \mathbf{u}\right\rangle_{\partial B(R)} .
$$

It is sufficient to consider the following approximation of the energy bilinear form in order to construct one term exterior approximation of the solution to the contact problem

$$
a(\rho ; \mathbf{u}, \mathbf{u}):=a(0 ; \mathbf{u}, \mathbf{u})+\rho^{2} b(\mathbf{u}, \mathbf{u}) .
$$

Denote by $\mathbf{H}_{\Gamma_{u}}^{1}(\Omega)=\left\{\mathbf{v} \in \mathbf{H}^{1}(\Omega) \mid \mathbf{v}=0\right.$ on $\left.\Gamma_{u}\right\}$ the Sobolev space, and let $K$ be the convex cone

$$
K=\left\{\mathbf{v} \in \mathbf{H}_{\Gamma_{u}}^{1}(\Omega) \mid v_{n} \geq 0 \text { on } \Gamma_{c}\right\} .
$$

Recall that the following variational inequality furnishes the weak solutions to our contact problem in $\Omega(\rho)$

$$
\mathbf{u} \in K: a(\rho ; \mathbf{u}, \mathbf{u}-\mathbf{v}) \geq L(\mathbf{v}-\mathbf{u}) \forall \mathbf{v} \in K .
$$

Taking into account the approximation (42) and using abstract results on the differentiability of metric projection onto the polyhedric convex sets in Dirichlet space [16] we have the following result.

Theorem 2. For $\rho$ sufficiently small we have on $\Omega(R)$ the following expansion of the solution $\mathbf{u}$ with respect to the parameter $\rho$ at $0+$,

$$
\mathbf{u}=\mathbf{u}_{0}+\rho^{2} \mathbf{q}+o\left(\rho^{2}\right) \text { in } \mathbf{H}^{1}(\Omega(R)),
$$

where the topological derivative $\mathbf{q}$ of the solution $\mathbf{u}$ to the contact problem is given by the unique solution of the following variational inequality

$$
\mathbf{q} \in \mathscr{S}_{K}(\mathbf{u}): a(0 ; \mathbf{q}, \mathbf{v}-\mathbf{q})+b(\mathbf{u}, \mathbf{v}-\mathbf{q}) \geq 0 \forall \mathbf{v} \in \mathscr{S}_{K}(\mathbf{u}),
$$

where

$$
\mathscr{S}_{K}(\mathbf{u})=\left\{\mathbf{v} \in \mathbf{H}_{\Gamma_{u}}^{1}(\Omega) \mid v_{n} \leq 0 \text { on } \Xi(\mathbf{u}), a(0 ; \mathbf{u}, \mathbf{v})=0\right\} .
$$

The coincidence set $\Xi(\mathbf{u})=\left\{\mathbf{x} \in \Gamma_{c} \mid u_{n}(\mathbf{x})=0\right\}$ is well defined [16] for any $\mathbf{u} \in \mathbf{H}^{1}(\Omega)$, and $\mathbf{u}_{0} \in K$ is the solution of (44) for $\rho=0$. 


\section{Complex Variable Method}

In order to find an exact form of the Steklov-Poincaré operator in plane elasticity we need an analytic form of the solution for the elasticity system in the ring, with general displacement condition on the outer boundary and traction free inner boundary, parameterized by the (small) inner radius $\rho$. Let us assume for simplicity that the center of the ring lies at origin of the coordinate system, and take polar coordinates $(r, \theta)$ with $\mathbf{e}_{r}$ pointing outwards and $\mathbf{e}_{\theta}$ perpendicularly in the counter-clockwise direction. Then the displacement on the outer boundary $r=R$ may be given in the form of a Fourier series

$$
2 \mu\left(u_{r}+i u_{\theta}\right)=\sum_{k=-\infty}^{k=+\infty} U_{k} e^{i k \theta} .
$$

The regularity condition for the boundary data translate into some inequalities for coefficients $U_{k}$, as will be made precise later.

The solution in the ring must be compared with the solution in the full circle, so we will have to construct it as well. Probably the best tool for obtaining both exact solutions is the complex variable method, described in [11]. It states that for plane domains with one hole these solutions have the form

$$
\begin{aligned}
\sigma_{r r}-i \sigma_{r \theta} & =2 \Re \phi^{\prime}-e^{2 i \theta}\left(\bar{z} \phi^{\prime \prime}+\psi^{\prime}\right), \\
\sigma_{r r}+i \sigma_{\theta \theta} & =4 \Re \phi^{\prime}, \\
2 \mu\left(u_{r}+i u_{\theta}\right) & =e^{-i \theta}\left(\kappa \phi-z \bar{\phi}^{\prime}-\bar{\psi}\right),
\end{aligned}
$$

where $\phi, \psi$ are given by complex series

$$
\begin{aligned}
& \phi=A \log (z)+\sum_{k=-\infty}^{k=+\infty} a_{k} z^{k}, \\
& \psi=-\kappa \bar{A} \log (z)+\sum_{k=-\infty}^{k=+\infty} b_{k} z^{k} .
\end{aligned}
$$

Here $\mu$ is the Lame constant, $v$ is the Poisson ratio, $\kappa=3-4 v$ in the plain strain case, and $\kappa=(3-v) /(1+v)$ for plane stress.

Now we can substitute displacement condition for $r=R$ into

$$
\begin{aligned}
2 \mu\left(u_{r}+i u_{\theta}\right) & =2 \kappa \operatorname{Ar} \log (r) \frac{1}{z}-\bar{A} \frac{1}{r} z+ \\
& +\sum_{p=-\infty}^{p=+\infty}\left[\kappa r a_{p+1}-(1-p) \bar{a}_{1-p} r^{-2 p+1}-\bar{b}_{-(p+1)} r^{-2 p-1}\right] z^{p}
\end{aligned}
$$

and obtain the infinite system of linear equations

$$
\begin{gathered}
p=-1: 2 \kappa A r \log (r)+\left(\kappa a_{0}-\bar{b}_{0}\right)-2 \bar{a}_{2} r^{2}=U_{-1} \\
p=1:-\bar{A}+\kappa r^{2} a_{2}-\bar{b}_{-2} \frac{1}{r^{2}}=U_{1} \\
p \notin\{-1,1\}: \kappa r^{p+1} a_{p+1}-(1-p) \bar{a}_{1-p} r^{-p+1}-\bar{b}_{-(p+1)} r^{-(p+1)}=U_{p} .
\end{gathered}
$$


The traction-free condition

$$
\sigma . \mathbf{e}_{r}=\left[\sigma_{r r}, \sigma_{r \theta}\right]^{\top}
$$

on some circle means $\sigma_{r r}=\sigma_{r \theta}=0$. Hence, assuming $r:=\rho$, we have another infinite system

$$
\begin{gathered}
p=-1: 2 A+2 \bar{a}_{2} r^{2}+2 \frac{1}{r^{2}} b_{-2}=0 \\
p=1:(\kappa+1) \frac{1}{r^{2}} \bar{A}=0 \\
p \notin\{-1,1\}:(1+p) a_{p+1}+\bar{a}_{1-p} r^{-2 p}+\frac{1}{r^{2}} b_{p-1}=0 .
\end{gathered}
$$

Denote $d_{0}=\kappa a_{0}-\bar{b}_{0}$ since $a_{0}, b_{0}$ appear only in this combination. Using (52) we may recover the solution for the full circle. Because in this case the singularities must vanish, we have $b_{-k}=a_{-k}=A=0$ for $k=1,2, \ldots$ and comparing the same powers of $r$ :

$$
\begin{aligned}
& d_{0}^{0}=U_{-1}+\frac{2}{\kappa} \bar{U}_{1}, \quad \Re a_{1}^{0}=\frac{1}{(\kappa-1) R} \mathfrak{N} U_{0}, \quad \mathfrak{I} a_{1}^{0}=\frac{1}{(\kappa+1) R} \mathfrak{I} U_{0} \\
& a_{k}^{0}=\frac{1}{\kappa R^{k}} U_{k-1}, \quad b_{k}^{0}=-\frac{1}{R^{k}}\left[(k+2) \frac{1}{\kappa} U_{k+1}+\bar{U}_{-(k+1)}\right], \quad k>1 .
\end{aligned}
$$

Now let us repeat the same procedure for the ring. Now the singularities may be present, because 0 does not belong to the domain. Hence, from (52) for $r=R$ and (54) for $r=\rho$ we obtain $A=0$ and the formulas

$$
\begin{array}{ll}
d_{0}=A_{-1}+\frac{2 R^{4}}{\kappa R^{4}+\rho^{4}} \bar{U}_{1}, & a_{2}=\frac{R^{2}}{\kappa R^{4}+\rho^{4}} U_{1} \\
\Re a_{1}=\frac{R}{(\kappa-1) R^{2}+2 \rho^{2}} \Re U_{0}, & \mathfrak{I} a_{1}=\frac{1}{\kappa+1} \mathfrak{I} A_{0} \\
b_{-1}=-\frac{2 \rho^{2} R}{(\kappa-1) R^{2}+2 \rho^{2}} \mathfrak{N} U_{0}, & b_{-2}=-\frac{\rho^{4} R^{2}}{\kappa R^{4}+\rho^{4}} \bar{U}_{1}
\end{array}
$$

The rest of the coefficients will be computed later. However, we may at this stage compare the results with known solutions for the uniformly stretched circle or ring obtained in another way. In such a case $U_{0}=2 \mu u_{r}(R)$ does not vanish and, for the full circle, $\psi=0, \phi=a_{1}^{0} z$ with

$$
a_{1}^{0}=\frac{2 \mu}{(\kappa-1) R} u_{r}(R) \text {. }
$$

For the ring we have $\phi=a_{1} z, \psi=b_{-1} \frac{1}{z}$ where

$$
a_{1}=\frac{1}{(\kappa-1)+2 \rho^{2}} 2 \mu u_{R}(1), \quad b_{-1}=-\frac{2 \rho^{2}}{(\kappa-1)+2 \rho^{2}} 2 \mu u_{R}(1) .
$$

After substitutions we obtain, in both cases, the same results as given in [7]. Similarly the comparison with the solution for the ring with displacement conditions on both boundaries, obtained in [4] also using complex method, confirms the correctness of the formulas. 
There remains to compute the rest of the coefficients $a_{k}, b_{k}$ for the case of the ring. Taking $p=-k, k=2,3, \ldots$ in conditions on both boundaries gives the system

$$
\begin{gathered}
\kappa a_{-(k-1)} R^{-(k-1)}-(k+1) \bar{a}_{k+1} R^{k+1}-\bar{b}_{k-1} R^{k-1}=U_{-k} \\
-(k-1) a_{-(k-1)} \rho^{2}+\bar{a}_{k+1} \rho^{2(k+1)}+b_{-(k+1)}=0,
\end{gathered}
$$

while $p=+k, k=2,3, \ldots$ results in

$$
\begin{aligned}
\kappa a_{k+1} R^{k+1}+(k-1) \bar{a}_{-(k-1)} R^{-(k-1)}-\bar{b}_{-(k+1)} R^{-(k+1)} & =U_{k} \\
(k+1) a_{k+1} \rho^{2(k+1)}+\bar{a}_{-(k-1)} \rho^{2}+b_{k-1} \rho^{2 k} & =0 .
\end{aligned}
$$

These systems may be represented in a recursive form, convenient for numerical computations and further analysis. Namely,

$$
S_{k}(\rho) \cdot\left[\begin{array}{l}
a_{k+1} \\
b_{k-1}
\end{array}\right]=\left[\begin{array}{c}
U_{k} \\
\bar{U}_{-k}
\end{array}\right]
$$

where $S_{k}$ has entries

$$
\begin{aligned}
& S_{k}(\rho)_{11}=\kappa R^{k+1}-\left(k^{2}-1\right) R^{1-k} \rho^{2 k}+k^{2} R^{-(k+1)} \rho^{2(k+1)} \\
& S_{k}(\rho)_{12}=-(k-1)\left(R^{1-k} \rho^{2(k-1)}-R^{-(k+1)} \rho^{2 k}\right) \\
& S_{k}(\rho)_{21}=-(k+1)\left(R^{k+1}+\kappa R^{1-k} \rho^{2 k}\right) \\
& S_{k}(\rho)_{22}=-R^{k-1}-\kappa R^{1-k} \rho^{2(k-1)}
\end{aligned}
$$

as well as

$$
\left[\begin{array}{l}
a_{-(k-1)} \\
b_{-(k+1)}
\end{array}\right]=T_{k}(\rho) \cdot\left[\begin{array}{l}
\bar{a}_{k+1} \\
\bar{b}_{k-1}
\end{array}\right],
$$

where

$$
T_{k}(\rho)=\left[\begin{array}{ll}
-(k+1) \rho^{2 k} & ,-\rho^{2(k-1)} \\
-k^{2} \rho^{2(k+1)} & ,-(k-1) \rho^{2 k}
\end{array}\right] .
$$

In fact the formulas 63, 61) are correct also for $k=0,1$ and in the limit $\rho \longrightarrow 0+$, but the derivation must separate these cases.

Thus, for given $k>1$ and using some initial $a_{k}, b_{k}$ obtained earlier, we may first compute $a_{k+1}, b_{k-1}$ using (61) and then $a_{-(k-1)}, b_{-(k+1)}$ from 63.

We may now use the above results for the asymptotic analysis of the solution. To simplify the formulas, we assume $R=1$, which means only rescaling and does not diminish generality (in general case $\rho$ would be replaced by $\rho / R$ ). Then by direct computation we get the following bounds for the differences between the coefficients on the full circle and the ring. For the initial values of $k$ they read

$$
\begin{aligned}
& d_{0}-d_{0}^{0}=-\rho^{4} \frac{2}{\kappa\left(\kappa R^{4}+\rho^{4}\right)} \bar{U}_{1} \\
& a_{1}-a_{1}^{0}=-\rho^{2} \frac{2}{(\kappa-1) R\left((\kappa-1) R^{2}+2 \rho^{2}\right)} \Re U_{0} \\
& a_{2}-a_{2}^{0}=-\rho^{4} \frac{1}{\kappa R^{2}\left(\kappa R^{4}+\rho^{4}\right)} U_{1}
\end{aligned}
$$


and for higher values

$$
\left|a_{3}-a_{3}^{0}\right| \leq \Lambda\left(\left|U_{2}\right| \rho^{4}+\left|U_{-2}\right| \rho^{2}\right)
$$

and for $k=4,5, \ldots$

$$
\left|a_{k}-a_{k}^{0}\right| \leq \Lambda\left(\left|U_{k-1}\right| \rho^{3(k-1) / 2}+\left|U_{1-k}\right| \rho^{3(k-2) / 2}\right)
$$

where the exponent $k / 2$ has been used to counteract the growth of $k^{2}$ in terms like $k^{2} \rho^{k / 2}$. Similarly

$$
\left|b_{1}-b_{1}^{0}\right| \leq \Lambda\left(\left|U_{2}\right| \rho^{4}+\left|U_{-2}\right| \rho^{2}\right)
$$

and for $k=2,3, \ldots$

$$
\left|b_{k}-b_{k}^{0}\right| \leq \Lambda\left(\left|U_{k+1}\right| \rho^{3(k+1) / 2}+\left|U_{-(k+1)}\right| \rho^{3 k / 2}\right) .
$$

From relation (63) we get further estimates

$$
\begin{aligned}
& \left|a_{-k}\right| \leq \Lambda \rho^{2 k}\left(\left|U_{k+1}\right|+\left|U_{-(k+1)}\right|\right), \quad k=1,2, \ldots \\
& \left|b_{-k}\right| \leq \Lambda \rho^{2(k-1)}\left(\left|U_{k-1}\right|+\left|U_{1-k}\right|\right), \quad k=3,4, \ldots
\end{aligned}
$$

Here $\Lambda$ is a constant independent from $\rho$ and $U_{i}$. Observe that the corrections proportional to $\rho^{2}$ are present only in $a_{1}, b_{1}, a_{3}, b_{-1}, a_{-1}$. The rest is of the order at least $O\left(\rho^{3}\right)$ (in fact $O\left(\rho^{4}\right)$ ).

These estimates may be translated into the following theorem concerning the solution of the elasticity system in the ring.

Theorem 3. The condition

$$
\|\mathbf{u}\|_{\mathbf{H}^{1 / 2}(\partial B(R))} \leq \Lambda_{0}
$$

which in terms of $U_{i}$ means

$$
\sum_{k=-\infty}^{k=+\infty} \sqrt{1+k^{2}}\left|U_{k}\right|^{2} \leq \Lambda_{0}
$$

ensures that the expression for elastic energy concentrated in the ring splits into the one corresponding to the full circle, correction proportional to $\rho^{2}$ and the rest, which is uniformly of the order $\Lambda_{0} \rho^{3}$.

\subsection{Numerical Illustration}

We shall show two solutions corresponding to different boundary conditions on the outer boundary, obtained using the representations derived above in terms of (in these particular cases finite) complex series.

Rugby-like deformation. Let us take $u_{r}=s_{0} \cos ^{2} \theta=\frac{1}{2} s_{0}+\frac{1}{2} s_{0} \cos 2 \theta$. Hence

$$
\left[U_{k}, k \in \mathbb{Z}\right]=\left[\ldots, \frac{1}{2} \mu s_{0}, 0, U_{0}=\mu s_{0}, 0, \frac{1}{2} \mu s_{0}, \ldots\right] .
$$



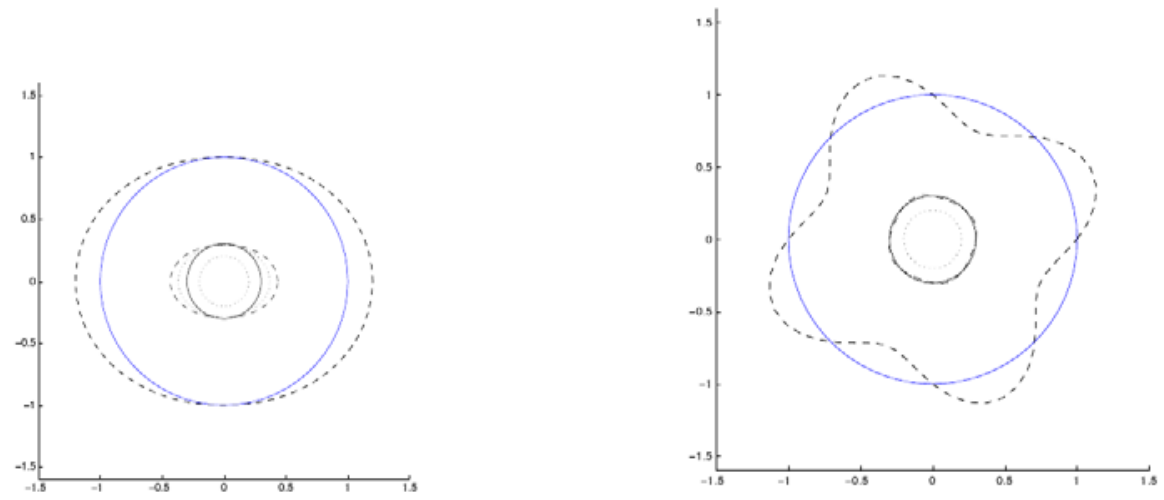

Fig. 1. Rugby-like and bubble-like distortions
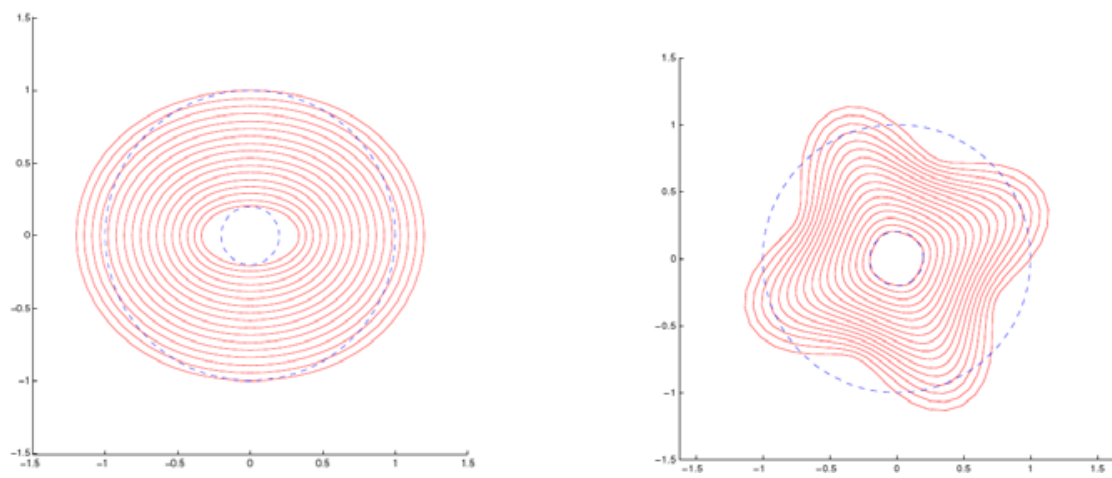

Fig. 2. The pattern of distortions for both experiments

The resulting distortion for size of the internal hole $\rho=0.2$ at the radius $r=0.3$ are shown in Fig. 1 (solid line - undeformed, dashed - deformed ring, dotted - deformed ball):

Bubble-like deformation. Now we take $u_{r}=s_{0} \sin 4 \theta$. Hence

$$
\left[U_{k}, k \in \mathbb{Z}\right]=\left[\ldots, \mu s_{0} i, 0,0,0, A_{0}=0,0,0,0,-\mu s_{0} i, \ldots\right] .
$$

The resulting distortions for $\rho=0.2$ and $r=0.3$ shows also Fig. 1. using the same types of lines.

In the second numerical experiment - bubble - only $U_{-4}$ and $U_{4}$ were nonzero, which means that the difference between positions of the contour $r=0.3$ for full circle and the ring should behave like $\rho^{6}$. In the first experiment it should be $\rho^{2}$, i.e. the influence of boundary condition should vanish quicker. The deformations for $\rho=0.2$ and several intermediate radii (dashed - undeformed, solid - deformed contours) are visible in Fig.2 and they confirm this observation. 


\section{Correction Term for Steklov-Poincaré Operator}

The elastic energy contained in the ring has the form

$$
2 \mathscr{E}(\rho, R)=\int_{C(\rho, R)} \sigma\left(u_{\rho}\right): \varepsilon\left(u_{\rho}\right) d x=\int_{\Gamma_{R}} u_{\rho} \sigma\left(u_{\rho}\right) \cdot n d s .
$$

Since $u_{\rho}=u$ on $\Gamma_{R}$

$$
2 \mathscr{E}(\rho, R)=\int_{\Gamma_{R}} u \sigma\left(u_{\rho}\right) \cdot n d s .
$$

Now $\sigma\left(u_{\rho}\right)$ is in fact of the form $\sigma\left(u_{\rho}\right)=\sigma_{\rho}(u)$, because $u_{\rho}=u$ on $\Gamma_{R}$, which means that $u_{\rho}=u_{\rho}(u)$. If we split $\sigma_{\rho}$ into

$$
\sigma_{\rho}(u)=\sigma^{0}+\rho^{2} \sigma^{1}(u)+O\left(\rho^{4}\right)
$$

then

$$
2 \mathscr{E}(\rho, R)=2 \mathscr{E}(0, R)+\rho^{2} \int_{\Gamma_{R}} u \sigma^{1}(u) . n d s+O\left(\rho^{4}\right) .
$$

Thus finding $\mathscr{A}_{1}$ reduces to computing $\sigma^{1}(u)$. From (49), 50) we know that $\sigma_{\rho}(u)$ is a linear function of infinite vectors $a=\left[a_{k}, k \in \mathbb{Z}\right], b=\left[b_{k}, k \in \mathbb{Z}\right]$, while $\sigma^{0}(u)$ is the same function of $a^{0}, b^{0}$. Here $a^{0}, b^{0}$ are computed for $B(R)$, while $a, b$ correspond to $C(\rho, R)$. In order to obtain $\sigma^{1}(u)$ it is enough to express $a, b$ as

$$
a=a^{0}+\rho^{2} a^{1}+O\left(\rho^{4}\right), \quad b=b^{0}+\rho^{2} b^{1}+O\left(\rho^{4}\right)
$$

because then

$$
\sigma^{1}(u)=\sigma^{1}\left(a^{1}, b^{1}\right) .
$$

In addition, the only nonzero terms in $a^{1}, b^{1}$ are $a_{3}^{1}, a_{1}^{1}, a_{-1}^{1}, b_{-1}^{1}, b_{1}^{1}$.

Taking into account that $A=0$ in (50) for our problem,

$$
\phi=\phi^{0}+\rho^{2} \phi^{1}+O\left(\rho^{4}\right), \quad \psi=\psi^{0}+\rho^{2} \psi^{1}+O\left(\rho^{4}\right)
$$

where

$$
\phi^{1}=a_{-1}^{1} \frac{1}{z}+a_{1}^{1} z+a_{3}^{1} z^{3}, \quad \psi^{1}=b_{-1}^{1} \frac{1}{z}+b_{1}^{1} z .
$$

Using formulas derived in preceding section, we may explicitly compute the coefficients appearing in 82 .

$$
\begin{gathered}
a_{-1}^{1}=-\bar{b}_{1}^{0}, \quad a_{3}^{1}=\frac{1}{\kappa R^{4}} b_{1}^{0}, \quad b_{1}^{1}=\frac{3+\kappa^{2}}{\kappa R^{2}} b_{1}^{0}, \\
a_{1}^{1}=-\frac{2}{(\kappa-1) R^{2}} \Re a_{1}^{0}, \quad b_{-1}^{1}=-2 \Re a_{1}^{0} .
\end{gathered}
$$

As is obvious from earlier calculations, only $U_{0}, U_{2}, U_{-2}$ will contribute to these corrections, Since

$$
U_{k}=\frac{\mu}{\pi} \int_{0}^{2 \pi}\left(u_{r}+i u_{\theta}\right) e^{-i k \theta} d \theta
$$


as well as

$$
u_{r}+i u_{\theta}=\left(u_{1}+i u_{2}\right) e^{-i \theta}
$$

then

$$
\begin{aligned}
U_{0} & =\frac{\mu}{\pi} \int_{0}^{2 \pi}\left(u_{1}+i u_{2}\right) e^{-i \theta} d \theta \\
U_{2} & =\frac{\mu}{\pi} \int_{0}^{2 \pi}\left(u_{1}+i u_{2}\right) e^{-3 i \theta} d \theta \\
U_{-2} & =\frac{\mu}{\pi} \int_{0}^{2 \pi}\left(u_{1}+i u_{2}\right) e^{+i \theta} d \theta .
\end{aligned}
$$

After collecting all formulas we obtain the final expression

$$
\begin{aligned}
\int_{\Gamma_{R}} u^{\top} \sigma^{1}(u) . n d s= & \frac{1}{R^{2}}\left[\frac{2(\kappa-2)}{(\kappa-1)^{2}}\left(\Re U_{0}\right)^{2}-(\kappa+1)\left|U_{-2}\right|^{2}\right. \\
& \left.-\frac{9(\kappa+1)}{\kappa^{2}}\left|U_{2}\right|^{2}-\frac{6(\kappa+1)}{\kappa} \Re\left(U_{2} U_{-2}\right)\right] .
\end{aligned}
$$

From 86 it follows that

$$
\begin{aligned}
\Re U_{0} & =\frac{\mu}{\pi} \int_{0}^{2 \pi}\left(u_{1} \cos \theta+u_{2} \sin \theta\right) d \theta \\
U_{2} & =\frac{\mu}{\pi} \int_{0}^{2 \pi}\left(u_{1} \cos 3 \theta+u_{2} \sin 3 \theta\right) d \theta+i \frac{\mu}{\pi} \int_{0}^{2 \pi}\left(u_{2} \cos 3 \theta-u_{1} \sin 3 \theta\right) d \theta \\
U_{-2} & =\frac{\mu}{\pi} \int_{0}^{2 \pi}\left(u_{1} \cos \theta-u_{2} \sin \theta\right) d \theta+i \frac{\mu}{\pi} \int_{0}^{2 \pi}\left(u_{2} \cos \theta+u_{1} \sin \theta\right) d \theta
\end{aligned}
$$

Here values of displacements are taken as $u_{i}(R \cos \theta, R \sin \theta)$. After discretization these integrals constitute weighted sums of values of $u_{i}$ at certain points on $\Gamma_{R}$. If we assume piecewise linear approximation over triangles, then it is well known that

$$
u_{i}^{h}(x)=x^{\top}\left[\begin{array}{lll}
x_{1}^{1} & x_{2}^{1} & 1 \\
x_{1}^{2} & x_{2}^{2} & 1 \\
x_{1}^{3} & x_{2}^{3} & 1
\end{array}\right]^{-1} \quad U_{i}^{h}=x^{\top} M^{-1} U_{i}^{h}
$$

and

$$
x^{\top} M^{-1} U_{i}^{h}=\left(M^{-\top} x\right)^{\top} U_{i}^{h}=c^{\top} U_{i}^{h}
$$

where $u_{i}^{h}(x)$ is a value of the approximation of $u_{i}$ at a point $x$ inside the triangle defined by vertices $x^{1}, x^{2}, x^{3}$ and $U_{i}^{h}$ is a vector of the values of $u_{i}^{h}$ at these vertices. Observe that $c$ is a vector of weights with which nodal values enter into the expression for $u_{i}^{h}(x)$. 
Let now $U^{h}=\left[u_{1}^{h 1}, u_{2}^{h 1}, \ldots, u_{1}^{h K}, u_{2}^{h K}\right]^{\top}$ be a vector of nodal values of $u^{h}$ for the global triangulation. Then we may write down the following formulae

$$
\begin{array}{lll}
\frac{\mu}{\pi} \int_{0}^{2 \pi} u_{1} \cos \theta d \theta=c_{11}^{\top} U^{h} & \frac{\mu}{\pi} \int_{0}^{2 \pi} u_{2} \sin \theta d \theta=s_{21}^{\top} U^{h} \\
\frac{\mu}{\pi} \int_{0}^{2 \pi} u_{1} \cos 3 \theta d \theta=c_{13}^{\top} U^{h} & \frac{\mu}{\pi} \int_{0}^{2 \pi} u_{2} \sin 3 \theta d \theta=s_{23}^{\top} U^{h} \\
\frac{\mu}{\pi} \int_{0}^{2 \pi} u_{1} \sin \theta d \theta=s_{11}^{\top} U^{h} & \frac{\mu}{\pi} \int_{0}^{2 \pi} u_{2} \cos \theta d \theta=c_{21}^{\top} U^{h} \\
\frac{\mu}{\pi} \int_{0}^{2 \pi} u_{1} \sin 3 \theta d \theta=s_{13}^{\top} U^{h} & \frac{\mu}{\pi} \int_{0}^{2 \pi} u_{2} \cos 3 \theta d \theta=c_{23}^{\top} U^{h} .
\end{array}
$$

Here $s_{i j}, c_{i j}$ are sparse vectors of weights with which nodal values of $u$ enter into appropriate integrals. In this notation

$$
\begin{aligned}
\left(\Re U_{0}\right)^{2}= & \left\|\left(c_{11}+s_{21}\right)^{\top} U^{h}\right\|^{2} \\
\left|U_{2}\right|^{2}= & \left\|\left(c_{13}+s_{23}\right)^{\top} U^{h}\right\|^{2}+\left\|\left(c_{23}-s_{13}\right)^{\top} U^{h}\right\|^{2} \\
\left|U_{-2}\right|^{2}= & \left\|\left(c_{11}-s_{21}\right)^{\top} U^{h}\right\|^{2}+\left\|\left(c_{21}+s_{11}\right)^{\top} U^{h}\right\|^{2} \\
\Re\left(U_{2} U_{-2}\right)= & \left(U^{h}\right)^{\top}\left(c_{13}+s_{23}\right)\left(c_{11}-s_{21}\right) U^{h} \\
& -\left(U^{h}\right)^{\top}\left(c_{23}-s_{13}\right)\left(c_{21}+s_{11}\right) U^{h} .
\end{aligned}
$$

Taking into account 87 we may conclude that the first term in the correction of energy is a well defined quadratic form. Similar, only more complicated expressions may be obtained for further asymptotics corresponding to $\rho^{4}$.

\section{References}

1. Ammari, H.: Polarization and Moment Tensors: with Applications to Inverse Problems and Effective Medium Theory. Applied Mathematical Sciences Series, vol. 162. Springer, New York (2007)

2. Eschenauer, H.A., Kobelev, V.V., Schumacher, A.: Bubble method for topology and shape optimization of structures. Struct. Optimiz. 8, 42-51 (1994)

3. Garreau, S., Guillaume, Ph., Masmoudi, M.: The topological asymptotic for PDE systems: the elasticity case. SIAM Journal on Control and Optimization 39, 1756-1778 (2001)

4. Gross, W.A.: The second fundamental problem of elasticity applied to a plane circular ring. Zeitschrift für Angewandte Mathematik und Physik 8, 71-73 (1957)

5. Hlaváček, I., Novotny, A.A., Sokołowski, J., Żochowski, A.: Energy change in elastic solids due to a spherical or circular cavity, considering uncertain input data. JOTA (2009) (in press)

6. Il'in, A.M.: Matching of Asymptotic Expansions of Solutions of Boundary Value Problems. Translations of Mathematical Monographs, vol. 102. AMS (1992)

7. Kachanov, M., Shafiro, B., Tsukrov, I.: Handbook of Elasticity Solutions. Kluwer Academic Publishers, Dordrecht (2003)

8. Lewinski, T., Sokołowski, J.: Energy change due to the appearance of cavities in elastic solids. Int. J. Solids Struct. 40, 1765-1803 (2003)

9. Masmoudi, M.: The toplogical asymptotic. In: Glowinski, R., Kawarada, H., Periaux, J. (eds.) Computational Methods for Control Applications, Gakuto (2002) 
10. Mazja, W.G., Nazarov, S.A., Plamenevskii, B.A.: Asymptotic theory of elliptic boundary value problems in singularly perturbed domains, vol. 1, 2. Birkhäuser Verlag, Basel (2000)

11. Muskhelishvili, N.I.: Some Basic Problems on the Mathematical Theory of Elasticity. Noordhoff (1952)

12. Nazarov, S.A., Sokołowski, J.: Asymptotic analysis of shape functionals. Journal de Mathématiques pures et appliquées 82, 125-196 (2003)

13. Nečas, J., Hlaváček, I.: Mathematical Theory of Elastic and Elasto-plastic Bodies: An Introduction. Elsevier, Amsterdam (1981)

14. Novotny, A.A., Feijóo, R.A., Padra, C., Taroco, E.A.: Topological sensitivity analysis. Computer Methods in Applied Mechanics and Engineering 192, 803-829 (2003)

15. Novotny, A.A., Feijóo, R.A., Padra, C., Taroco, E.A.: Topological sensitivity analysis for three-dimensional linear elasticity problems. Computer Methods in Applied Mechanics and Engineering (to appear)

16. Rao, M., Sokołowski, J.: Tangent sets in Banach spaces and applications to variational inequalities. Tech. Rep. Les prépublications de l'Institut Élie Cartan 42/2000 (2000)

17. Sokołowski, J., Zolésio, J.-P.: Introduction to Shape Optimization. Shape Sensitivity Analysis. Springer, Heidelberg (1992)

18. Sokołowski, J., Żochowski, A.: On topological derivative in shape optimization. SIAM Journal on Control and Optimization 37(4), 1251-1272 (1999)

19. Sokołowski, J., Żochowski, A.: Topological derivatives of shape functionals for elasticity systems. Mechanics of Structures and Machines 29, 333-351 (2001)

20. Sokołowski, J., Żochowski, A.: Optimality conditions for simultaneous topology and shape optimization. SIAM Journal on Control and Optimization 42, 1198-1221 (2003)

21. Sokołowski, J., Żochowski, A.: Modelling of topological derivatives for contact problems. Numerische Mathematik 102(1), 145-179 (2005) 